\title{
KNOWLEDGE GENERATION THROUGH HUMAN-CENTERED INFORMATION VISUALIZATION
}

\author{
Katja Einsfeld ${ }^{1}$, Achim Ebert ${ }^{1}$, Andreas Kerren ${ }^{* 2}$, and Matthias Deller ${ }^{3}$ \\ ${ }^{1}$ Computer Science Department, Visualization Lab, University of Kaiserslautern, \\ PO Box 3049, D-67653 Kaiserslautern, Germany \\ ${ }^{2}$ School of Mathematics and Systems Engineering (MSI), Växjö University, \\ Vejdes Plats 7, SE-351 95 Växjö, Sweden \\ ${ }^{3}$ Competence Center Human-Centered Visualization (HCV), German Research Center for Artificial Intelligence (DFKI), \\ Trippstadter Strasse 122, D-67663, Kaiserslautern, Germany.
}

\begin{abstract}
One important intention of human-centered information visualization is to represent huge amounts of abstract data in a visual representation that allows even users from foreign application domains to interact with the visualization, to understand the underlying data, and finally, to gain new, application-related knowledge. The visualization will help experts as well as non-experts to link previously or isolated knowledge-items in their mental map with new insights.

Our approach explicitly supports the process of linking knowledge-items with three concepts. At first, the representation of data items in an ontology categorizes and relates them. Secondly, the use of various visualization techniques visually correlates isolated items by graph-structures, layout, attachment, integration, or hyperlink techniques. Thirdly, the intensive use of visual metaphors relates a known source domain to a less known target domain. In order to realize a scenario of these concepts, we developed a visual interface for non-experts to maintain complex wastewater treatment plants. This domain-specific application is used to give our concepts a meaningful background.
\end{abstract}

Keywords: Information visualization, semantic data, human-centered visualization, human-computer interaction, wastewater treatment

\section{Introduction}

While Information Visualization (InfoVis) research often concentrates on visualization techniques for one isolated data type, some applications have to deal with huge amounts of abstract and unstructured data of various data types. This might be one of the reasons why innovative information visualization techniques are only reluctantly used in some application domains. A human-centered perspective on information visualization $[1,2]$ can help to get-among other things - an overview of isolated visualization techniques and to structure them in such a way

\footnotetext{
*Corresponding author. Email: kerren@ acm.org
}

that they can be implemented in real-world applications in a usable and modular manner. Therefore, one of the main challenges is to integrate individual InfoVis techniques in a way that semantic relations between them are intuitively clear even to non-expert users (this is also a goal of the emerging field of Visual Analytics [3]).

In this article, we describe several techniques and concepts we have implemented in a framework in order to meet this challenge. After a short discussion on our domain background in the next Subsection 1.1, we present information visualization techniques for the analysis of huge amounts of complex process data (Section 2). Here, we have developed, integrated and made use of techniques that allow to relate individual techniques to each other. For example, we utilized the advantages of virtual reality and integrated information visualization techniques into this approach. By employing ontologies and semantic techniques, we were able to order, structure, and relate the heterogeneous input data into more meaningful information.

In Section 3, we describe how our implementation meets human needs. The application of the information visualization reference model [4] allows us to react on user interaction in a modular and context-sensitive way. With the help of additional concepts derived from HumanComputer Interaction (HCI), such as visual metaphors [5], we created a syntheses of all these elements. An application that provides intuitive access to information allows to gain knowledge on complex processes and smoothly reacts on user interaction. Thus, it provides an appropriate context-sensitive view.

\subsection{Domain Background}

Our work is part of the research project KOMPLETT ([6]; "komplett" is German for "complete"). In this project, a consortium of partners from universities and industry develops a small innovative WasteWater Treatment Plant (WWTP) which can be utilized in arid areas for decentralized water recycling purposes. WWTPs constitute an innovative and strong scenario for applying and testing our interactive visualization concepts. 
The overall idea of the WWTP is to collect the wastewater of a small housing area or hotel in two separate fractions - black water and gray water-and to treat these fractions in two separate processes. Gray water can be recycled up to drinking water and treated black water can be used, for example, as irrigation water or service water for toilets. From the perspective of the plant engineers, the challenge is to apply state of the art wastewater treatment techniques like physical treatment, biological treatment (membranes), and chemical treatment to separated water fractions and-compared to ordinary WWTPs-really small amounts of water. Moreover, engineers had to find out how to place all needed devices and tubes in the limited area of, for example, four containers.

Plants like this will likely be located in remote areas and cannot be maintained on a daily basis by experts. Thus, the caretaker of a housing area or hotel has to do the daily maintenance work. On the other hand, the technical equipment and the processes in the plant are quite complex. Furthermore, the traditionally available information for WWTPs is unstructured, unconnected, heterogeneous, and available in diverse data types and media depending on manufacturers and previous maintainers. This makes it even more difficult for non-experts to understand, handle and maintain such a plant.

\subsection{Visualization Framework}

The goal of our visualization tool is to support educational, monitoring and optimization tasks of those technically complex plants in an intuitive and user-adaptive way. Bertelsen and Nielsen [7] studied different theoretical frameworks that offer ways of analyzing the work at a modern wastewater treatment plant. They found that, in the case of WWTPs, deviations and situated action limits the formalization in CSCW-design. They concluded that "Design approaches enabling technical tailoring based on strong modularization and encapsulation [...] may help". Consequently, from the perspective of information visualization, the challenge of the project is to ensure the usability of the plant for non-experts by developing a visualization system that bridges the gap between the unstructured data and the non-expert by creating intuitive visual interfaces and human-centered interaction possibilities.

Our framework is implemented in C++ and OpenGL. The functionality of the processes in the WWTP, the physical layout of the plant, links to measured data from the plant, and additional information on the plant are organized in an ontology that can be accessed by the application in order to visualize single items in their context. The process data acquired from the plant is stored in a database. In general, an ontology is a description of a specific domain of interest, including concepts, categories, entities, attributes, and interrelationships. In computer science, ontologies can be used for the retrieval of semantics (through the relations) or for automatic reasoning. A detailed explanation of ontologies can be found in the work of Uschold and Grüninger [8]. Ceccaroni et al. [9] made use of ontologies in the domain of WWTPs, and Bosca et al. [10] proposed a $3 \mathrm{D}$ visualization technique for ontologies.

\section{Information Visualization Techniques}

In this section, an overview of information visualization techniques for diverse data types available in our application is provided. This ranges from visualization techniques for large quantitative data sets to graph visualizations to the visualization of semantics and relations.

\subsection{Quantitative Process Data}

There are large quantities of process data in our application area, such as temperature, pressure, or nitrate concentration measured at the plant. Each value can be qualified with a timestamp and an item-ID. The item-ID is, with the help of the ontology, associated to its semantic context. For example, using the device which recorded an item-ID, we can identify its type and value (temperature, pressure, ... ), the physical and the logical part of the plant or process to which the measuring device belongs, available documentation for that device, and so on.

Systems that have to accomplish with huge amounts of data usually have to decide whether they display one detail view at a time (time multiplexing) or show all information in different parts of the screen (space multiplexing) simultaneously $[4,11,12]$. While space multiplexing can lead to cluttered views, users of time multiplexing techniques are in risk of losing the context. We propose for our concrete problem the use of animation as a solution to this space vs. time multiplexing dilemma. 3D and animation provide one possibility to visualize more information in a "natural" focus+context style in our special case. By choosing metaphors derived from common concepts of the wastewater treatment domain, a more intuitive and humancentered access to information can be accomplished.

Color scale is highly configurable in our framework. The interface designer can choose between various objects of a class hierarchy, e.g., two-color gradients, multi-color gradients, and gradients that consist of two or three individual gradients divided by one ore two thresholds. The default gradient we used consists of an dark-blue to lightblue two-color gradient in the lower $20 \%$, a dark-green to light-green to brown multi-color gradient in the middle, and a light-red to dark-red two-color gradient in the upper $20 \%$ of the value spectrum. This decision was taken by two considerations: the findings on color scales by Rheingans [13] and Light et al. [14] show that rainbow color scales are hard to interpret due to the fact that the order of multiple colors is not perceived preattentively and that various degrees of luminance improve the quality of a color scale. The second consideration was that the application area of process data monitoring and analysis often requires a qualitative interpretation of the data. To ensure a human-centered interface, we decided to separate the color scale with two thresholds 

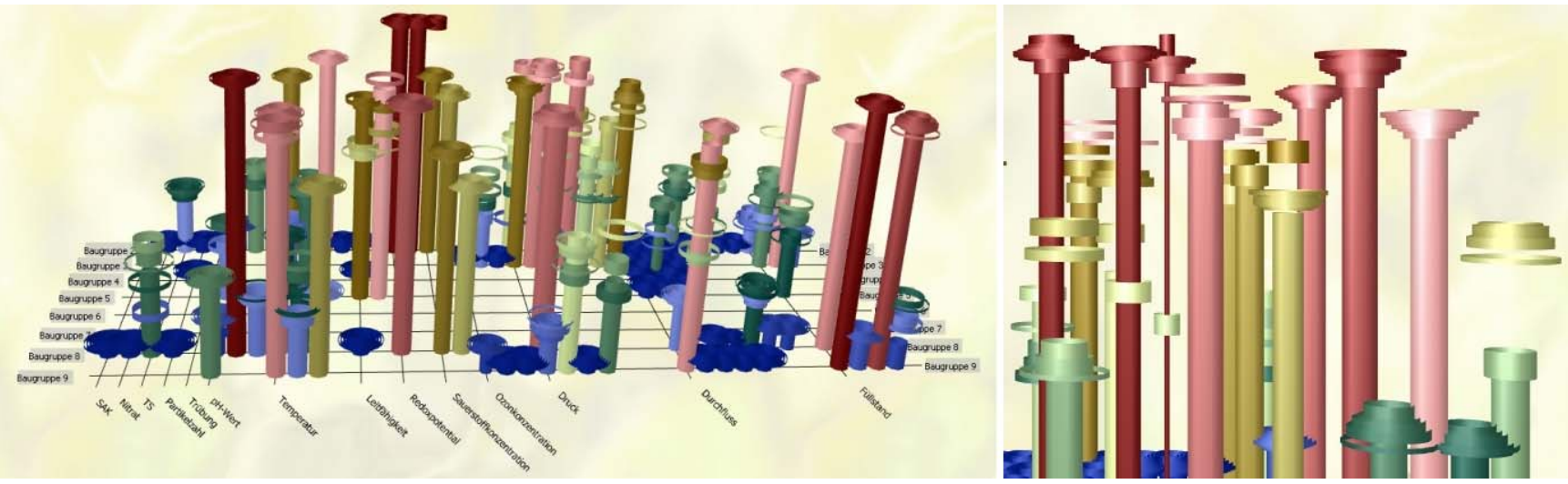

Figure 1. 4D Process Overview (left) and detail extract (right)
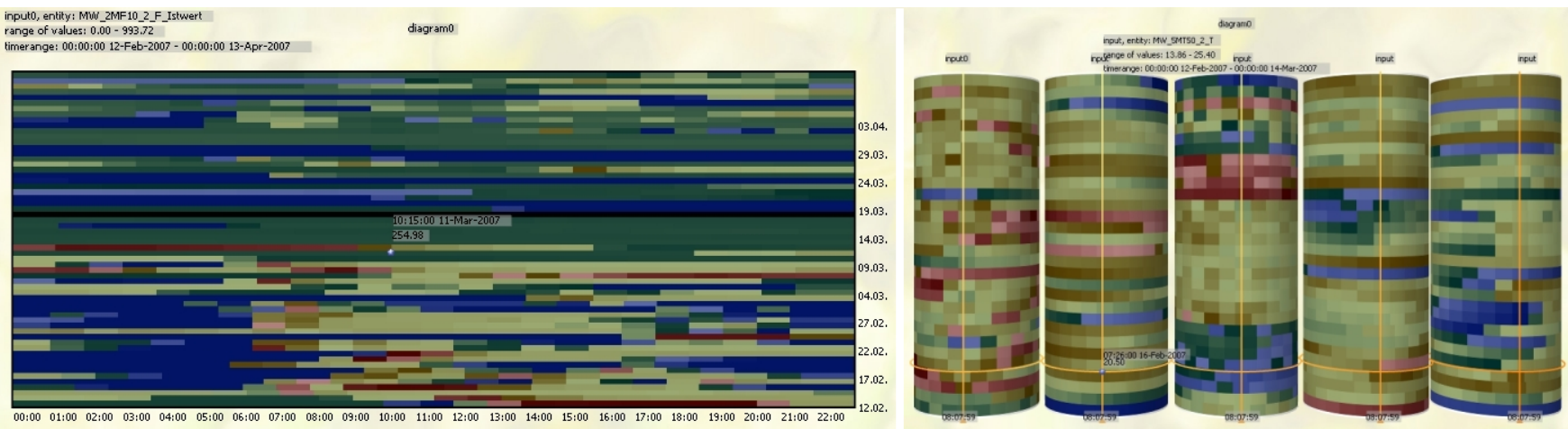

Figure 2. Color Plane (left) and Process Data Rolls (right)
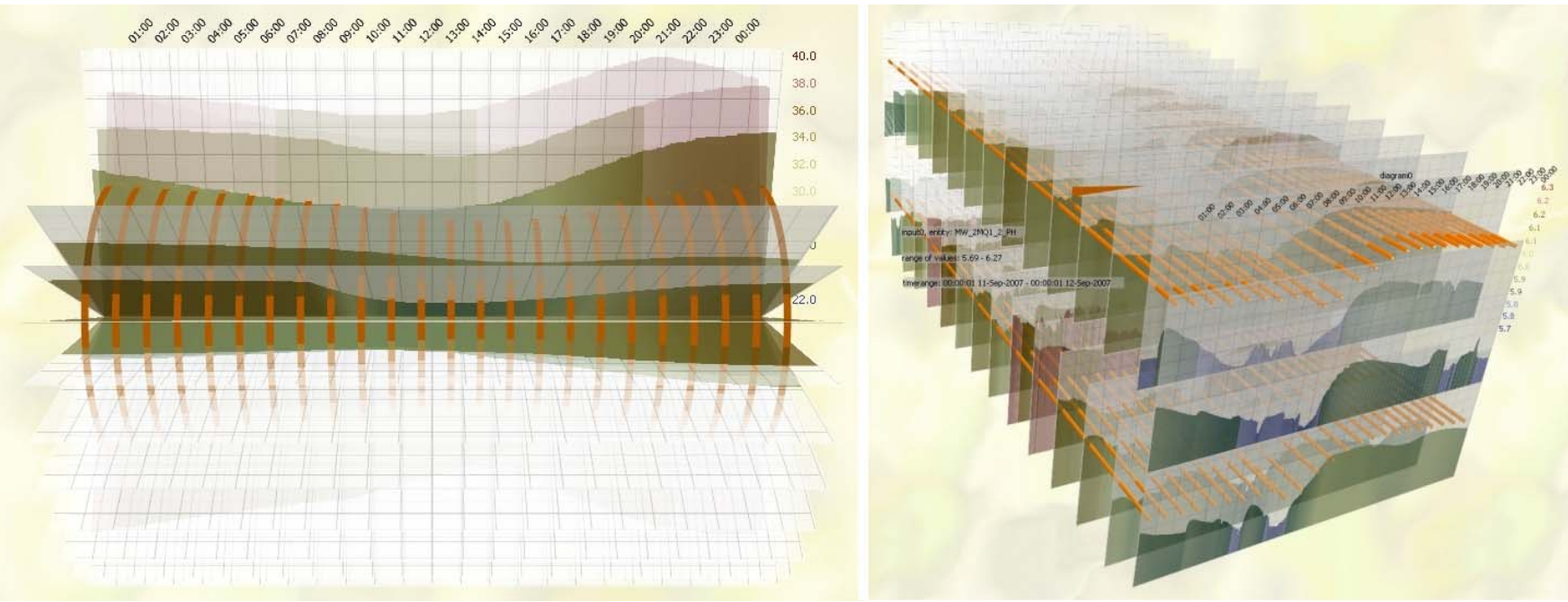

Figure 3. Rotary Diagram (left) and Conveyor Belt (right) 
in three visually distinctive parts to indicate that some values are precariously low, high, or in an acceptable range. All our diagrams use the same color scale that can be adjusted with a color scale widget. While the programmer has the possibility to modify the colors and the type of the color scale as described above, the user can only change the upper and lower threshold with the help of the widget.

The concept of process visualization in our framework is hierarchical as well as modular. There is a toolbox of metaphors for process data visualization from which the user can easily choose and combine the most appropriate metaphors, depending on current context and personal preferences. Some metaphors are used to gain overview over all parameters (cp. Figure 1); some are used to compare the behavior of selected parameters (cp. Figure 2); some are used to analyze the behavior of one parameter over time (cp. Figure 3); finally, there are also focus views for detailed analysis.

In order to get an overview over process data from the whole process, we developed a 4D (3D plus time) visualization with animated bars as shown in Figure 1. Each bar represents one parameter. The parameter values are mapped to the height of the bars and their color. The user can specify the time interval of interest. When the animation is started, a time slider moves from start-time to endtime, the values of the parameters change according to the current animation time, and the bars move up and down depending on the current value. The spatial order of the bars is determined by semantic information gained from the ontology: on the ground plane, the $\mathrm{x}$-axis lists the parameter types and the $y$-axis the physical plant parts. The items of both axes can be rotated in order to move other bars to the front or focus position. The thickness of bars represents the changing behavior of the values of that parameter, i.e., the result of the ratio deviation/averagevalue is mapped to the radius of the bars.

The right part of Figure 1 shows a detail view of this metaphor that helps to explain the additional history rings: the last five values before the current time are indicated with rings. The elevation (z-position) of the center of the rings as well as their colors indicate past values. To indicate the age of the data, rings are getting broader and less tall over time, using an intuitive visual "dissolving" metaphor. When animated, the history rings follow the corresponding bar as they move up and down. Their dynamic behavior reminds of the natural behavior of concentric waves on a water surface emanating from the center. This visual metaphor allows the user to preattentively distinguish increasing values (cone shape), decreasing values (upsidedown cone shape), constant values (compressed sphere), and fast changing values (stretched cone).

\subsubsection{Spotting Patterns and Comparing Parameters}

The Color Plane visualization of process data, shown in Figure 2 (left), is comparable to other well-known InfoVis approaches, like the technique presented by Kincaid and
Lam [15]. The idea is to map a value $v$ not to the y-axis, but to a color code only. Thus, the y-axis can be used to visualize a consecutive sequence of these colored bars. Color Planes visualize a value $v$ of one parameter in the time interval $i$ (e.g., four weeks) that are subdivided in $n$ equally sized intervals $j_{i, k}$ (e.g., days) from $k=1, \ldots, n$. Each of the intervals $j_{i, k}$ is represented by a single color-coded horizontal bar in the plane. Thus, the $\mathrm{x}$-axis shows the timeline from the start to the end of each interval $j_{i, k}$, while the $\mathrm{y}$-axis depicts the order of the $j_{i, k}$-intervals in the $i$ interval. Compared to traditional approaches where values are mapped to the y-axis, this technique visualizes more spacefilling and allows the user to discover common patterns or irregularities when comparing the intervals $j_{i, k}$.

We extended this approach by making use of the third dimension and animation in order to be able to compare multiple parameters: Figure 2 (right) shows our so-called Process Data Rolls. Each roll represents one parameter with its Color Plane mapped around a cylinder. The cylinders can simultaneously be rotated which brings a specific time of interest from an interval $j_{i, k}$ (e.g., evenings of all days) to the front. Due to the 3D shape of the cylinders, a natural and intuitive focus+context effect results from the perspective distortion. Positive user feedback inspired us to add vertical lines and horizontal rings that further improve the analysis of interrelated behavior across various parameters.

\subsubsection{Comparing Consecutive Intervals and Average Values}

Figure 3 shows two visual metaphors derived from daily life objects: the Rotary Diagram (left) and the Conveyor Belt (right). Using simple metaphors that are easy to interact with mostly results in an intuitive use. Both metaphors presented in Figure 3 visualize the value of one parameter subdivided in $n$ intervals $j_{i, k}$. In contrast to Color Planes, the traditional $y$-axis representation of process data is kept. Thus, users that prefer this representation can also find a suitable diagram type in our toolbox.

The time vs. space multiplexing dilemma is again solved with the help of 3D and animation: the semitransparent style of each diagram allows the human visual system to directly compare consecutive diagrams. The orange rings in the Rotary Diagram and the rods in the Conveyor Belt which represent the average value of all intervals $j_{i, k}$ in a specific time segment $i$ further enhance the comparability and the possibility to discover outliers.

\subsection{Graph-Based Data}

Data that can be structured in a graph-based form, i.e., in nodes and edges, is common in our application area: there are instructions, the flow direction of the wastewater through the plant, the hierarchical order of plant parts and technical devices, or the order of process steps. As the expert knowledge is stored in the form of an ontology, 

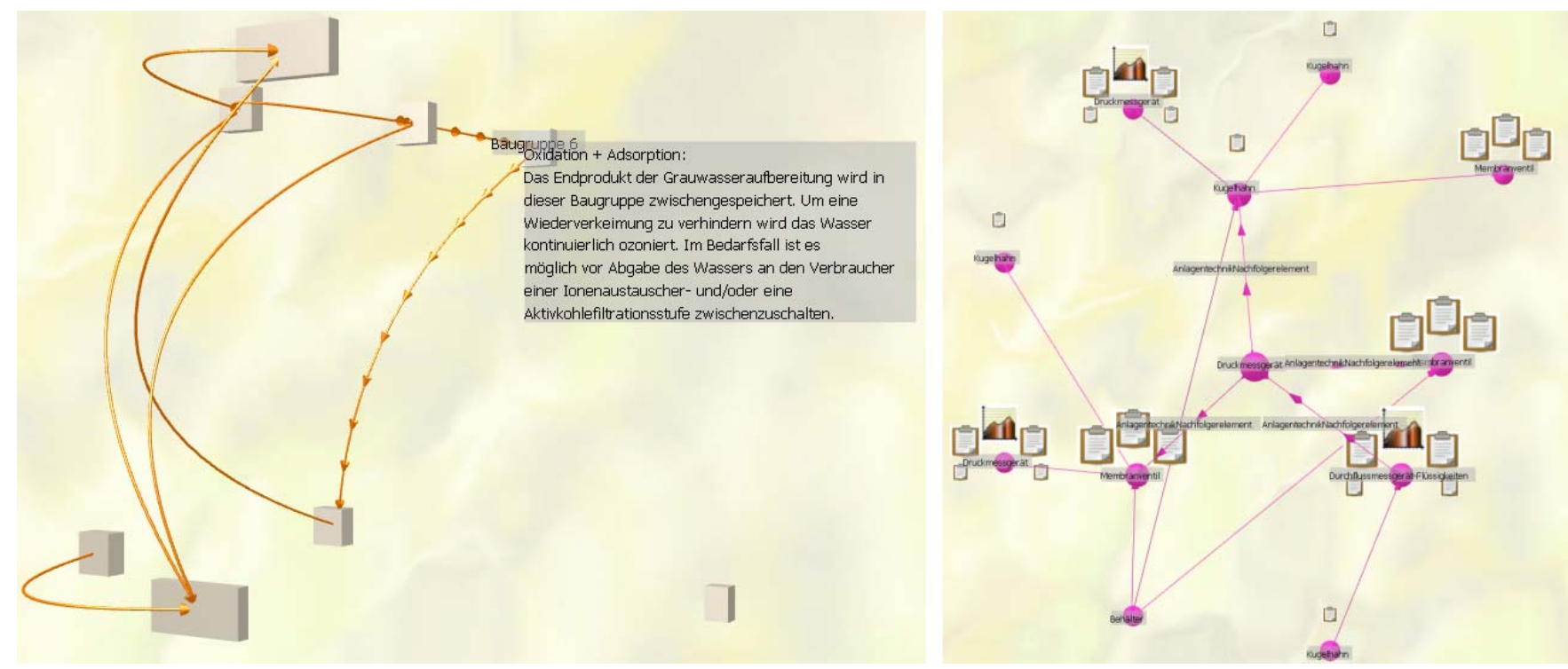

Figure 4. Visualization of graph-based data
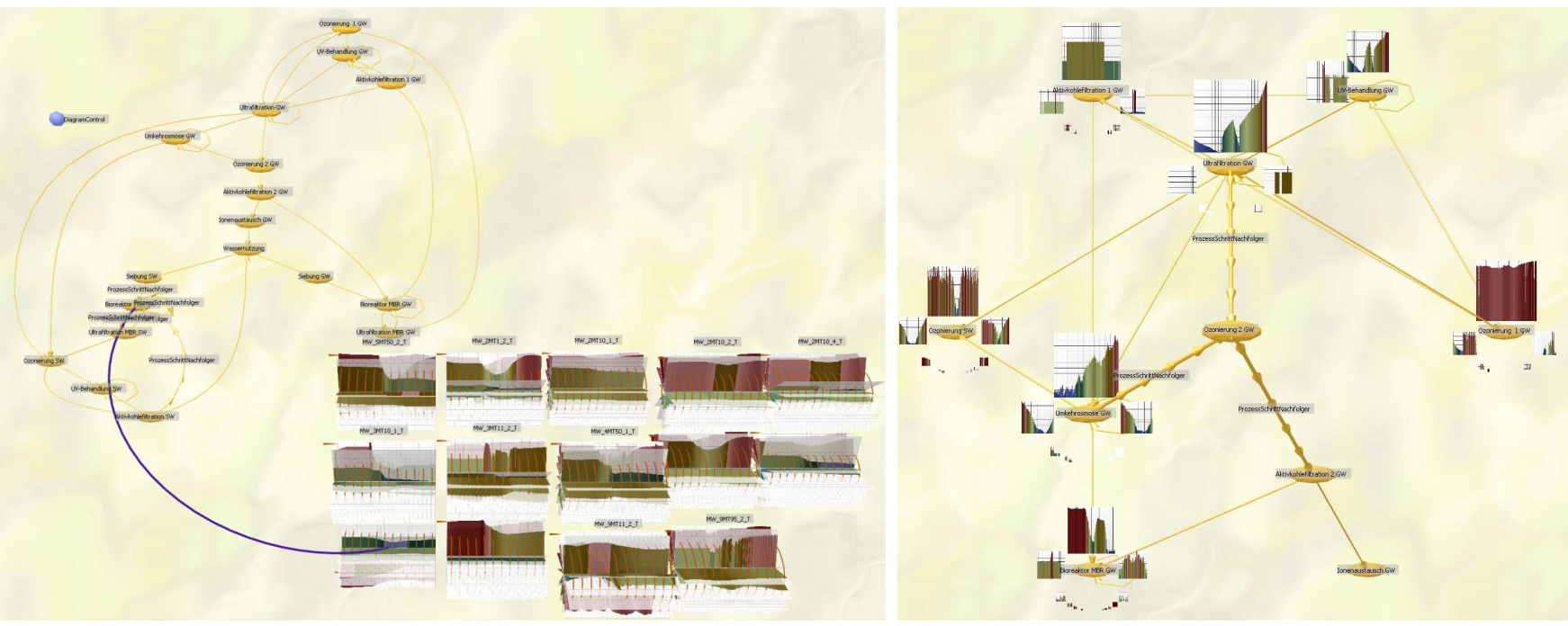

Figure 5. Side by side visualization (left) and integrated visualization with Focus Circles (right)

there are a manifold of possibilities to extract graph structures, such as class hierarchies, relations of instances of one class, or relations between instances from diverse classes depending on the current context.

In our application, we used various visualization approaches for graph-based data. Depending on their purpose, they vary in their degree of detail or overview, dynamics or fixed positions, abstractness or virtual realism, and 3D. Our framework architecture allows us to reuse some of the elements, e.g., the edges with animated arrows, in multiple graph types, see for example Figure 4.

Figure 5, left hand side, shows a 2D overview visualization of the process steps and their order in water-flow direction, which was generated with the help of the opensource library Graphviz [16]. On the right hand side of this figure, a 3D detail visualization of the process is visualized. The focus node is located in the front and center position, and the connected nodes are positioned in circles in a cone-like shape around the center. To change the current focus, the user can click on one of the nodes on the two circles, which smoothly moves the selected node to the focus position and draws connected nodes around it. This view allows a good perception of the focused node and its direct context. The user can switch between focus view and overview, triggering an animated transition. Both of these graph layouts calculate the position of the nodes dynamically depending on the graph structure.

Figure 4, left hand side, shows the visualization of wastewater flow (edges) through the plant parts (nodes). In this case, the positions of the nodes are fixed, as they re- 
flect the physical position of the plant parts. Compared to the real plant layout, the plant parts are visualized as abstract boxes and in reduced size to avoid occlusion of the flow direction indicators. The VR visualization of one plant part, as shown in Figure 7, can also be seen as a graph visualization because it visualizes elements of the plant and their connection via tubes. The purpose of this fixed node $3 \mathrm{D}$ visualization with a high degree of realism is, however, to promote the recognition of the physical plant and the interaction with it. In contrast, dynamic and abstract graph layouts are more useful for understanding and browsing complex processes.

\subsubsection{Chart-Based Diagrams in Graph-Based Context}

In applications with complex semantic contexts, it is beneficial for mental integration to visualize chart-based diagrams within their semantic context. This context can often be represented as an abstract graph structure or a 3D visualization of real-world geometry. Figure 5 shows two examples of integrating charts in graph structures. On the left hand side, an overview visualization of the WWTP-process steps is displayed which was generated from our ontology with the help of Graphviz. All temperature measurements of the process are visualized as small Rotary Diagrams in a separate area. These are moved to the front depending on the discrepancy between their current and their average value. In that way, the user can identify irregular behaviors at one glance. The semantic connection between the diagrams and their context in the graph is visually represented by connecting curves when either a diagram or a graph node is clicked, $\mathrm{cp}$. the next section on semantic data.

The right part of Figure 5 shows a focus view of the process. All parameters measured at a specific process step are visualized with the help of Focus Circles around the nodes. A Focus Circle moves the clicked element in an animated way around a circle to the top position where it has the largest magnification. Thus, the user can easily scroll through all charts of the process to look for irregularities. Other application areas of Focus Circles can be seen in Figure 4, right side, and Figure 6, where they are used with icons that allow to access additional information.

\subsection{Semantic Data}

Semantic data is data that explains the category, context, or meaning of other data. Semantic data can occur, for example, in the form of a structured set of variables that describe one data item (metadata) or in the form of numerous, diverse triples in the Resource Description Framework (RDF) model. As semantic data can be used to describe the categories or attributes of data items, it usually deals with heterogeneous, previously unstructured data of different type.

As indicated in Section 1, our application has to deal with huge amounts of heterogeneous, semantically struc-

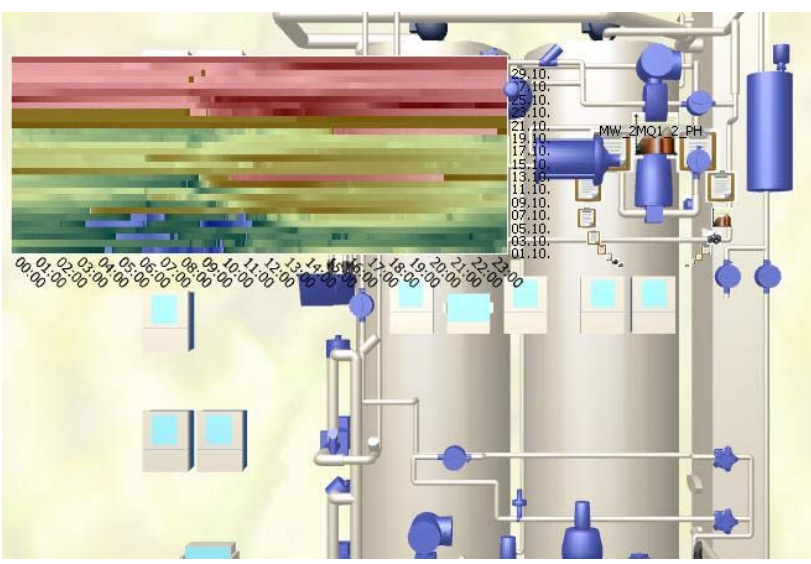

Figure 6. Accessing abstract data through virtual reality interfaces

tured data. Our domain experts, together with information scientists, have developed an ontology to categorize, describe and relate the information items (small isolated pieces of information) to each other. This ontology contains information about

- processes in the WWTP,

- technical components and their order in the process,

- measured data,

- documentation linked to the respective components,

- instructions for maintenance linked with involved plant parts and documentation, and

- diagnosis elements with proposed solutions for critical plant conditions.

This semantic information is exported in RDF-format and then imported in a database in order to be fast and uniformly accessible from various applications.

Figure 6 shows one example of visualizing semantic data. The available metadata for one technical component is organized as icons on a Focus Circle around the component (right upper corner of the screenshot). When the user clicks the icon in the top and focus position of the circle, the respective metadata is opened, i.e., a PDF document with manufacturer information, an image, a video, or a Color Plane with data from an process item is shown. Textual metadata can be attached to nodes in the form of transparent or semitransparent labels as indicated in Figure 4 (left). Also Figure 5 shows two possibilities of how semantic relations between diverse types of information can be visualized. On the left hand side of the image, the process-step nodes and the process data are organized in separated areas and connected with 3D edges when the user clicks one of the elements. On the right hand side, the process data is displayed within the Focus Circles. Another possibility to visualize semantic data in a human-centered way is to use the concept of modified virtual reality. 

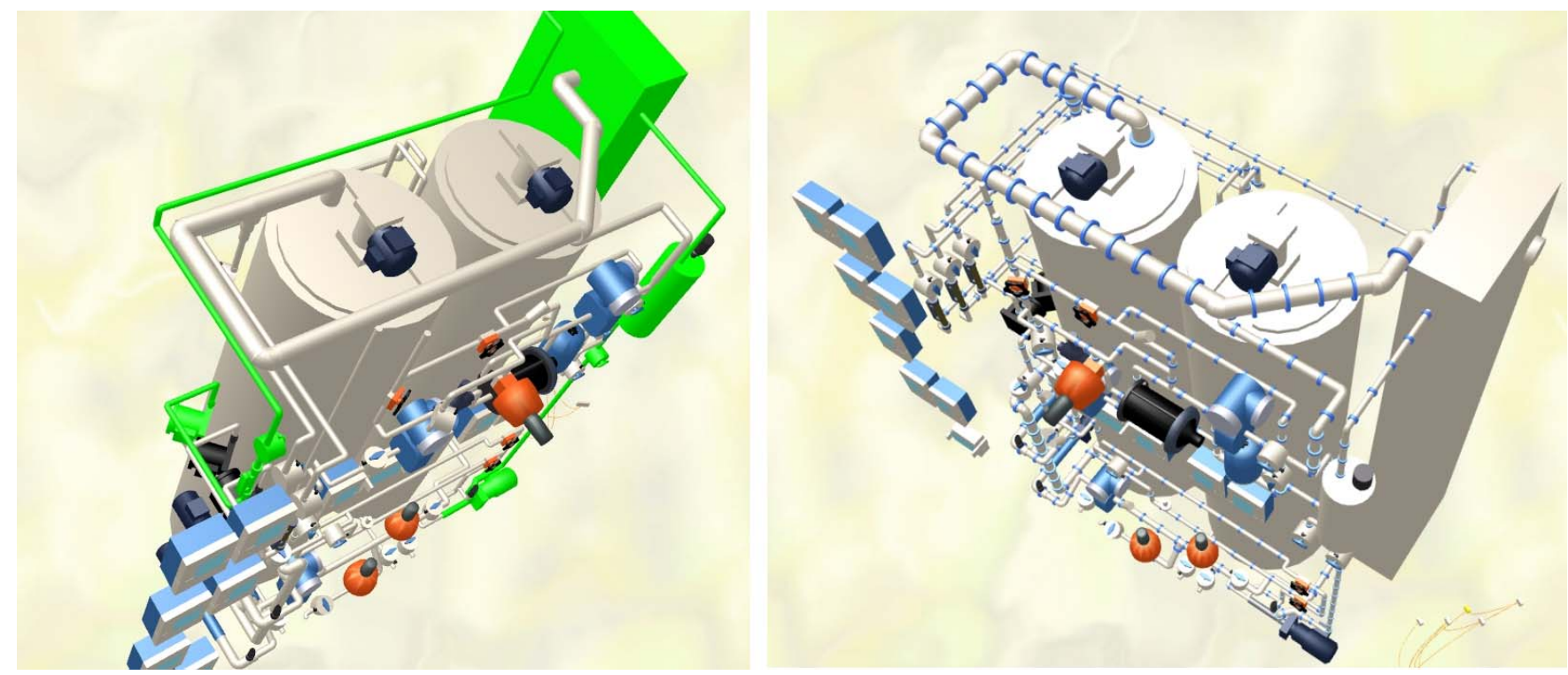

Figure 7. Modified virtual reality

\subsubsection{Modified Virtual Reality}

In a previous publication [17], we proposed a modified virtual reality as a technique to integrate and access information in the VR visualization of the physical application domain (in our case the WWTP). This is a human-centered way of visualization, because it bridges the gap between the physical plant and the available abstract information that non-experts usually have to face. The user perceives the information directly integrated into the item to which it belongs. Figure 7 shows two examples of direct information integration. On the left hand side, some of the objects are highlighted by using a signal color to indicate the connection between two components. This can be useful for error detection or educational purposes. On the right hand side of the image, the geometry of the tubes is modified with animated blue rings that move along the tubes, visualizing the flow direction of water or air in the tubes. Other possibilities of modified virtual reality are hierarchical extracts, animation (e.g., pulsing behavior), or attached icons (see Figure 6).

\subsection{Connections between Various Data Types}

There are many reasons why the visual representation of semantic connections between diverse data types and views results in more human-centered applications than the isolated visualization of single data types. In an informal evaluation we found that users seem to dislike switching between various views. They are reluctant to remember how to invoke single views and when to use them. They feel more comfortable with views that can integrate additional data on demand or connect them to additional visual representations of data needed in the current context.

We also found that visualizing individual data types in separated views does not help the user to sufficiently understand the semantic connections between these views. Without any visual connections, it is hard to understand the relations, for example, between the physical layout of the plant, the process data, and the visualization of process steps. Another argument for the visual representation of semantic connections is that it supports the human way of sensemaking. In order to understand new and complex situations, humans create mental maps by connecting and relating known facts with new ones [18].

Sections 2.2 and 2.3 already described techniques for integrating and visually connecting various data type visualizations in one view. The integration of chart-based diagrams in their graph-based semantic context adds additional information that enables the user to understand and interpret the chart depending on its context in the system [19]. We also discussed how diagrams can be integrated in the 3D visualization of our application in order to bridge the semantic gap between the physical layout of the plant and abstract data.

\subsubsection{Visual Integration vs. Hyperlinks of Connec- tions}

Another approach of the visual representation of connections between various data types implemented in our application is an architecture that is similar to a web browser: information from the ontology is dynamically layouted, i.e., depending on the context, semantically related information like diagrams, images, documents, or textual information is displayed in the same view, opened externally when the user clicks the item, or hyperlinked in another view. Hyperlinks are visualized as labels with semitransparent background in the affordance color (see Section 3.2). This approach is especially useful in contexts where textual information is of central importance. In our application area 


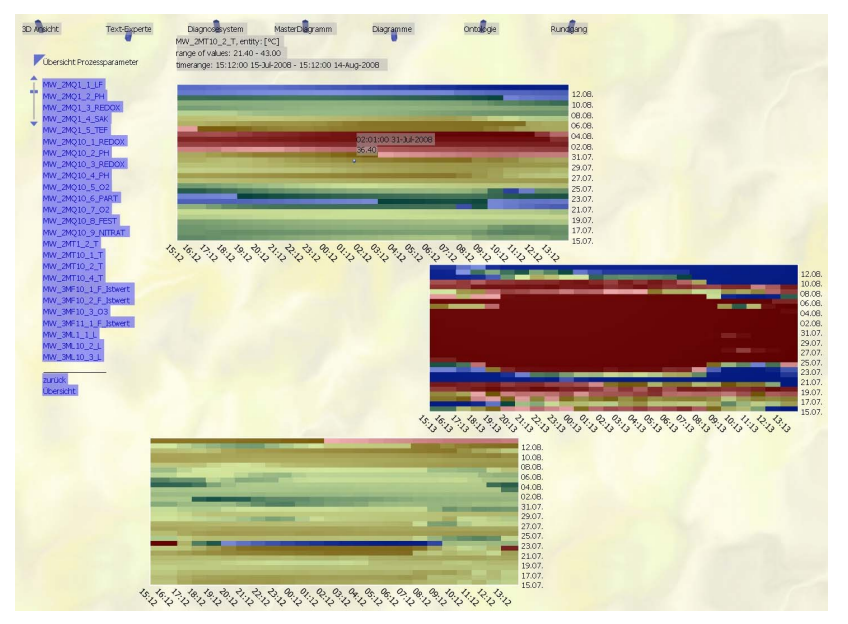

Figure 8. Browser-like architecture in the diagnosis system

there are at least two tasks where this kind of visualization can be deployed: the diagnosis system and the maintenance instructions.

In the diagnosis system, there are possibilities to browse information depending on its category (documents, process data, images, etc.) and to search the database with the help of textual search patterns. Moreover, there is a set of diagnosis situations that can be searched in order to understand the reasons for problematic situations and to get some suggestions for possible solutions together with additional information (e.g., manufacturer documents or expert contacts). If there are formal descriptions of diagnosis situations available in the ontology, these situations are checked automatically with the help of a parser implemented on the basis of the boost::spirit framework [20]. As described above, the textual information is dynamically layouted, and the additional information is either automatically integrated in the view, shown on demand, or hyperlinked in other views. Figure 8 shows a screenshot of the diagnosis system and the expert-knowledge database (knowledge from the ontology): process parameters are visualized as hyperlinks in a scroll list (left hand side), and the diagrams of the parameters appear when the user clicks on the hyperlinks.

Figure 9 shows two screenshots of the daily maintenance instructions the caretaker of the plant has to follow. The user can click through all the steps, which are dynamically layouted in separate views and linked together. If there is additional information available, such as a diagnosis, the caretaker has to validate process parameters, technical components of the plant, documentation, images or anything else that is linked, as described above. In the upper screenshot in Figure 9, the task of the caretaker is to validate the functionality of a measuring device. The device is shown as an automatically integrated picture. The chart with the past values of this device appears when the user clicks the link. The lower screenshot of Figure 9 shows the task of checking the functionality of a pump. In this in-

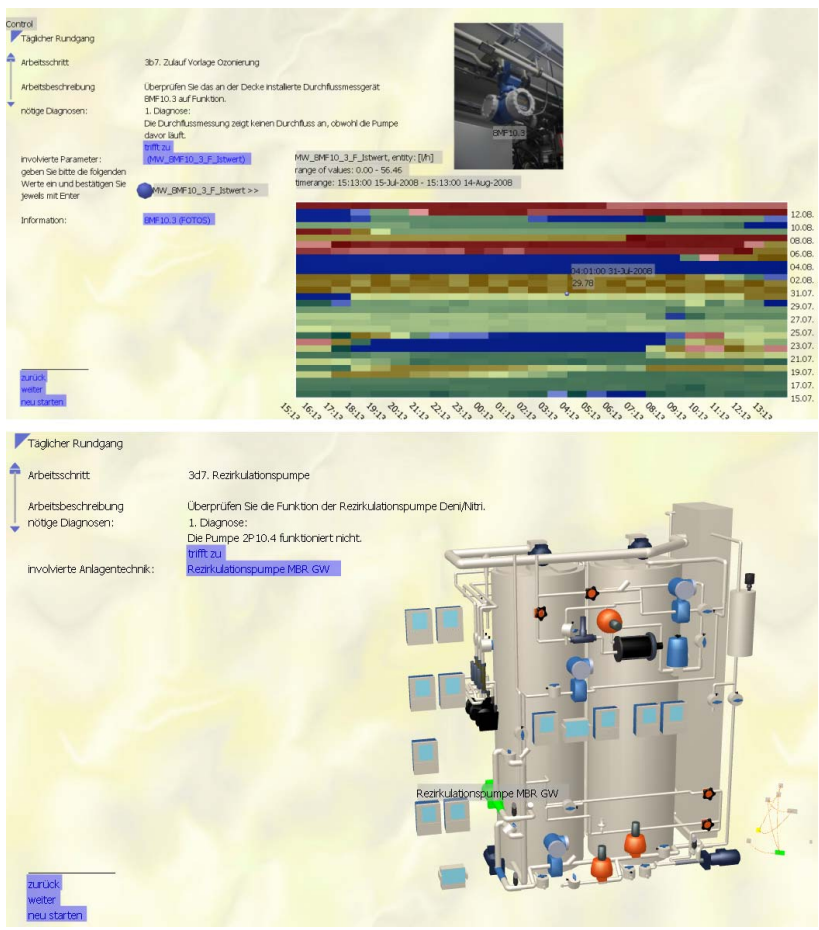

Figure 9. Browser-like architecture in the walk-through check

struction step, visual integration is utilized by animating the 3D plant part containing the highlighted pump to the outside of the overall plant structure.

The next Section 3 provides more details on the human-centered implementation of connections between different data types.

\section{Human-Centered Design Aspects}

Modern Process Control Systems (PCS; e.g., see web page [21]) support the staff during operation of wastewater plants, e.g., by automatization of control strategies and documentation of process data. But, the staff also depends on additional information like manuals, service instructions, technical maps, knowledge of colleagues, etc. Because of the high diversity and the large amount of information necessary to characterize and operate a complex wastewater treatment plant, it is important to have access to the information directly at the place of work. In addition, in our case not only experts need to operate the WWTP, but also non-experts have to shoulder some of the daily work.

To achieve these goals, it is firstly necessary to transform the available data into usable information, which is available at any location and at any time. Secondly, common PCS have been developed for expert users only, such making the monitoring and control of WWTPs a complex task. Therefore, in decentralized waste water treatment approaches, new concepts for the user interfaces are needed. They have to be tailored on the intended user group of nonexperts, thereby giving them the possibility to operate the 
WWTP without a time- and cost-intensive training. The visualization system has to provide as much information as possible to the user, simultaneously taking care of not overwhelming him or her and presenting the information as meaningfully as possible. This clearly points out the need for interactive, human-centered visualization metaphors.

\subsection{Translating Raw Data into Information}

The data state model developed by Chi [22] and described as information visualization reference model by Card et al. [4] can be seen as a kind of Model-View-Controller pattern (MVC) for InfoVis applications. According to Card et al., a data transformation firstly converts raw, abstract data into structured data tables. The structure used to represent the data has to be suitable for the chosen visualization. In a second step, a visual mapping is used to convert data to visual structures. Thirdly, view transformations convert these structures to specific views.

This model helps to separate the four layers and to isolate dependencies, which allows to easily reuse different parts of our implementation. Thus, the raw data can be restructured to create multiple visualizations of the data source, and the visual structures can be transformed to allow the user to gain various perspectives on the visualization, e.g., by using zoom, rotation or other operations for reorganizing or modifying the elements of a visualization.

From the perspective of interaction, applications implementing the InfoVis reference model have several advantages. The modular and reusable structure of the application allows to create flexible and context sensitive views that can be adapted to the current user needs. Thus, the user can interact with the application at three diverse levels: data transformation, visual mapping, and view transformation. This kind of interaction can easily be implemented and handled in a modular way, which leads to applications with numerous flexible interaction possibilities and less interaction constrains. In consequence, the user is not forced to understand what the machine is doing, but can choose between several intuitive visualization metaphors, depending on his/her current tasks and individual preferences.

As already indicated in Section 1, the raw data of our application consists of large amounts of complex heterogeneous data that are categorized, structured, and related in the form of an expert knowledge base in our ontology. This knowledge management is performed by domain experts and information scientists together with the help of the ontology editor Protégé [23]. Additional information in diverse media formats (process data in database tables, videos, images, documentation, 3D objects) is referenced from the ontology. The ontology is exported in RDFformat with the help of the RSSDB tool [24] and then imported into multiple database tables. In this way, the "raw data" is already pre-structured in order to be able to access the semantic information uniformly from any context.

At the user interaction layer of the data transformation step, the user chooses the visualization type (e.g., VR visualization, diagnosis system, or process data visualization) and calls for connected visualization items if necessary. The required process data is loaded from the database into Diagramlnput objects, where some preprocessing calculations are done, for example, the calculation of minimum and maximum values. Semantic data that is needed for the abstract graph visualization or any other graph operations is loaded in the CommonGraph data structure that stores node types, edge types, nodes, edges, and the connections between them. In other cases, semantic data will simply be loaded in associative containers that will, for example, store the ID of a technical component item as a key and the ID of information items available for that component as values. Moreover, there are specialized classes that can store special purpose information from the ontology.

In the user interaction layer of the visual mapping, the user can decide what type of process data visualization (e.g., Rotary Diagram or ColorPlane) he/she wants to map to the abstract data and what color scale to use for this mapping. He/she can also decide how to interact with the VR visualization (navigating through the hierarchy, using modified-virtual-reality techniques, etc.), how to navigate graph-based views (e.g., focused view or overview), or how to interact with the browser-like visualization for the diagnosis system or the maintenance instructions (call for linked information, follow links to other layouts).

At the user interaction layer of view transformations there are also multiple possibilities: our general concept of manipulating the $3 \mathrm{D}$ view is the thought-wizard-navigation metaphor described by Einsfeld et al. [25]. The idea of this metaphor is to relieve the user from the burden of moving and trying to orientate in 3D space and instead give $\mathrm{him} / \mathrm{her}$ the power to use simple gestures to move and manipulate the objects in front of him/her. There are possibilities to scale, translate, and rotate the objects either with mouse gestures or with navigation control tools. Ware et al. [26] found that the motion parallax depth cue is very important for the human perception of 3D. Due to this fact we added another interaction possibility - the shake metaphor-that is more easy to use than conventional rotation of objects with the mouse: with simple triggers (gestures or key presses) the user can "shake" objects, i.e., an object or scene will rotate within a small angle around the $\mathrm{x}$ - or $\mathrm{y}$-axis. This small movement provides the motion parallax depth cue and allows the user to perceive 3D quickly and without conscious effort.

The next section describes the advantages of visual mappings from structured data to visual metaphors if meaningful metaphors are used.

\subsection{Visual Metaphors}

Metaphors are used to compare a phenomenon $a$ of domain $d_{a}$ (the metaphor's target) to a phenomenon $b$ of domain $d_{b}$ (the metaphor's source). This helps humans to create a vivid mental map of $a$-especially in cases where domain $d_{a}$ is more abstract than domain $d_{b}$, or the individual is 
more familiar with domain $d_{b}$ than with domain $d_{a}$. Thus, metaphors help to extend knowledge about things we understand to new domains of experience.

A visual metaphor is a metaphor in which visual attributes of the source are used to facilitate the understanding of or the interaction with the target. The target does not necessarily need to have a visual representation. Thus, an abstract target can be mapped to a visual representation with the help of the visual attributes of the visual metaphor's target. The usefulness of visual metaphors in information visualization, e.g., for decision making, has also been emphasized by Görg et al. [19].

Carefully designed visual metaphors enable a more natural representation of abstract data that can intuitively be interpreted by domain experts and laymen, thereby resulting in a human-centered access to complex data. To be obvious to human beings it is required that the metaphor's source stems from domains that are well-known from the user's daily life and thus intuitively to understand. A visual metaphor is even more effective and intuitive in the human-centered sense if not only the visual attributes but also the interaction-related attributes of the source object are ascribed to the target object. This results in more coherent and convincing metaphors that are not only intuitive to understand, but also to interact with.

Risch [27] elaborates on the advantages of visual metaphors in information visualization. He states that "the degree of structural alignment of a particular visualization with one or more corresponding image schemas accounts for its perceived degree of intuitiveness". Image schemas are, according to Risch, "cognitively entrenched patterns of physical experience that theoretically serve as a key bridge between perception and cognition" and are, thus, ideal candidates for metaphor-sources. This is due to the fact that they "serve to structure and constrain abstract reasoning processes via metaphorical projection operations that are grounded in everyday cognitive and perceptual experiences".

In our application, we use multiple visual and navigation metaphors. Section 2.1 described some of the visual metaphors used for the visualization of abstract process data: the rings in the master diagram (Figure 1), Color Planes \& Process Data Rolls (Figure 2), and Rotary Diagram \& Conveyor Belt (Figure 3). Additionally, Section 3.1 mentioned the thought-wizard and shakenavigation metaphors. The VR view of the plant is rather an analogy than a metaphor as it presents the real physical layout of the plant in a very similar spacial layout in the visualization. According to Risch, analogies are, together with metaphors, one of the key aspects of human cognition.

Gibson [28] pointed out that affordances (visual items that are perceived as "i can do something with it") are important in the creation of user-centered interaction interfaces. The affordances of the metaphor's source are usually attached to its target. Thus, the user intuitively knows how to interact with the visual metaphor. In cases where this is not possible, we propose to apply an affordance color: a color that is used for control elements and any other items the user can interact with. While not being as intuitively understandable as natural affordances and requiring introductory information about the color, it nevertheless gives the user a preattentive cue about usable objects. Figure 6 shows how some of the technical components in the VR view in semantic-information mode are colored with the affordance color (in this case blue). When the user clicks one of the blue devices, a Focus Circle with icons appears. Through the icons, the plant documentation, diagrams for measured parameters, and possibilities to control the devices are accessible.

Section 2.4 already explained the importance of visual representations of semantic information for humancomputer interfaces. The semantic approach bridges the gap between previously unrelated data items; it supports the human way of sensemaking and thus helps to transform data to knowledge. Our application provides the user with context-information on demand, visually interrelates semantically related visual items, and supports the user with extensible semantic search possibilities.

\section{Evaluation and Comparison with other Approaches}

To confirm the benefits of our implementation, we did an evaluation with the involved parties, both domain experts and non-expert users (e.g., caretakers) of the system. Since most of the employed metaphors and techniques have no equivalent solutions in our application domain, we postponed a formal comparative evaluation in favor of an informal one done by the designated users of the system. We have presented our interfaces to a group of WWTP experts, who are used to work with conventional process control systems, and to a group of laymen in the domain. At the first glimpse, they were doubtful whether the new visual metaphors will really be applicable in their domain. However, after a closer inspection of the new visual functionalities, they quickly recognized the potential of our visualization and interaction metaphors. They also testified that they expect that the new metaphors should give non-experts the chance to easily handle a large portion of daily routine work. Our system is currently installed in a pilot plant at the Fraunhofer UMSICHT in Oberhausen, Germany [29]. This installation will give us the possibility to gather more data about the potential of the new metaphors in a real-world scenario.

As already mentioned, we have not yet conducted a formal evaluation of our approach. Moreover, we think that this formal step will be very difficult to accomplish. The reason for this is that we could only compare our system with existing PCS that are focused on domain experts as users. So our main target group, non-experts, will not be able to control a PCS in a reasonable time. Therefore, a comparative evaluation in this application domain is expected to be far less conclusive than a field test of the sys- 
tem. Outside the domain of waste water treatment, there are, however, some newer frameworks that integrate and connect diverse information visualization techniques in a real-world application. One typical example is the work of Jern et al. [30]. The authors use multiple windows with color-coded maps, scatterplots, parallel coordinates, and other well-known techniques to visualize sociological, economical and ecological data of Sweden. Compared to our approach, Jern's framework, which is in this respect similar to many other information visualization applications, utilizes the multiple-view concept. An excellent starting point for related work of coordinated and multiple view visualization techniques is the annual conference series on Coordinated \& Multiple Views in Exploratory Visualization (CMV) or the work of Roberts [31]. The possibilities to visually relate individual visualizations to each other is often not sufficient: instead of using brushing, glyphs or direct feedback of user interactions in all windows simultaneously, our approach offers intuitive possibilities of visual relations like spacial layout, attachment, integration, or hyperlinked visualizations.

There are even less examples that combine InfoVis techniques with real-world geometry visualization of the application area: Kirner et al. [32] placed visual elements, such as circle diagrams, bars, text elements, and metaphors like books, in a walk-through environment in order to visualize the behavior of visitors of a museum in the virtual analogon of the real environment. Although there is no formal evaluation available, their approach of placing information visualizations in a VR world seems to be less intuitive than our techniques of integrating information.

\section{Conclusion}

In our informal evaluation, we found that our visual metaphors help users to understand their meaning and interaction possibilities and, additionally, increase the joy of interacting with them. Especially 3D supports the creation of perspective focus+context views and its combination with transparency and/or animation allows the framework to visualize our application data intuitively. It also facilitates mental integration of data inside a naturally threedimensional context (the plant simulation) as well as visual comparison of datasets. Compared to common time or space multiplexing techniques, details and context remain perceivable. Animation enables the user to understand and keep track of changes, or to compare consecutive items. Moreover, the use of animation amplifies the impression of natural objects in $3 \mathrm{D}$ space which results in an immersive visualization and enables a more human-centered interaction with the application.

Besides these methods, we also explained how visual techniques, for example integrating chart-based diagrams into their (graph-based) semantic context, modified virtual reality, information integration, or browser-like visualization, can help to understand the complex semantic relations between isolated information items. The user can inter- pret the information item in its context, which leads to a deeper and more intuitive understanding of the whole information system and the interrelation of individual information items.

We used ontologies as a semantic data storage technique and Card's information visualization reference model to ensure the flexibility and natural user-interaction possibilities. Finally, we described how we integrated these techniques and concepts in our application in order to create a flexible and human-centered interface, intuitively usable by non-experts as well as experts of the domain.

\section{Acknowledgments}

This research is part of the interdisciplinary project KOMPLETT, which is supported by the German Federal Ministry of Education and Research (BMBF, grant number 02WD0685). We thank our colleagues from the Institute of Urban Water Management of the Technical University of Kaiserslautern for sharing their expert knowledge and work on the ontology.

\section{References}

[1] Kerren A, Ebert A, Meyer J, editors. HumanCentered Visualization Environments, GI-Dagstuhl Research Seminar, Dagstuhl Castle, Germany, March 5-8, 2006, Revised Lectures. vol. 4417 of Lecture Notes in Computer Science. Springer; 2007.

[2] Kerren A, Stasko JT, Fekete JD, North C, editors. Information Visualization, Human-Centered Issues and Perspectives. vol. 4950 of Lecture Notes in Computer Science. Springer; 2008.

[3] Keim D, Andrienko G, Fekete JD, Görg C, Kohlhammer J, Melançon G. Visual Analytics: Definition, Process, and Challenges. In: Kerren A, Stasko JT, Fekete JD, North C, editors. Information Visualization. vol. 4950 of Lecture Notes in Computer Science. Springer; 2008. p. 154-175.

[4] Card SK, Mackinlay JD, Shneiderman B. Readings in Information Visualization: Using Vision to Think. Academic Press; 1999.

[5] Catarci T, Costabile MF, Matera M. Visual metaphors for interacting with databases. SIGCHI Bull. 1995;27(2):15-17.

[6] Project KOMPLETT; 2009 [accessed March 2009]. http: / / www.komplett-projekt.de/ index.php?id=3601\&L=en.

[7] Bertelsen OW, Nielsen C. Dynamics in wastewater treatment: a framework for understanding formal constructs in complex technical settings. In: Proceedings of the Sixth European conference on 
Computer supported cooperative work. Norwell, MA, USA: Kluwer Academic Publishers; 1999. p. 277290.

[8] Uschold M, Grüninger M. Ontologies: Principles, methods and applications. Knowledge Engineering Review Cambridge University Press. 1996;11:93136.

[9] Ceccaroni L, Cortés U, Sànchez-Marrè M. OntoWEDSS: augmenting environmental decisionsupport systems with ontologies. Environmental Modelling and Software. 2004;19(9):785-797.

[10] Bosca A, Bonino D, Pellegrino P. OntoSphere: More than a 3D Ontology Visualization Tool. In: Proceedings of SWAP 2005, the 2nd Italian Semantic Web Workshop, Trento, Italy, December 14-16, 2005, CEUR Workshop Proceedings; 2005. .

[11] Fekete JD, Plaisant C. Interactive Information Visualization of a Million Items. In: INFOVIS '02: Proceedings of the IEEE Symposium on Information Visualization (InfoVis'02). Washington, DC, USA: IEEE Computer Society; 2002. p. 117.

[12] Hart D, Tudoreanu ME. Visualization Channels: Time Multiplexing on a Display. In: Hamza MH, editor. VIIP '01: Proceedings of the Conference on Visualization, Imaging and Image Processing. ACTA Press; 2001. p. 95-100.

[13] Rheingans P. Task-Based Color Scale Design. In: Proceedings of Applied Image and Pattern Recognition. SPIE; 1999. p. 35-43. citeseer. ist.psu. edu/rheingans99taskbased.html.

[14] Light A, Bartlein PJ. The End of the Rainbow? Color Schemes for Improved Data Graphics. EOS Transactions. 2004 Oct;85:385-391.

[15] Kincaid R, Lam H. Line graph explorer: scalable display of line graphs using Focus+Context. In: AVI '06: Proceedings of the working conference on Advanced visual interfaces. New York, NY, USA: ACM Press; 2006. p. 404-411.

[16] Graphviz - Free Open Source Graph Visualization Software; 2009 [accessed March 2009]. http:// www.graphviz.org/.

[17] Einsfeld K, Ebert A, Wölle J. Modified Virtual Reality for Intuitive Semantic Information Visualization. In: IV '08: Proceedings of the 12th International Conference on Information Visualisation. Washington, DC, USA: IEEE Computer Society; 2008. p. 515-520.

[18] Cosgrove D. Mappings. Reaktion Books; 1999.
[19] Görg C, Pohl M, Qeli E, Xu K. Visual Representations. In: Kerren A, Ebert A, Meyer J, editors. Human-Centered Visualization Environments. vol. 4417 of Lecture Notes in Computer Science. Springer; 2006. p. 163-230.

[20] boost::spirit - An object oriented recursive descent parser framework implemented using template metaprogramming techniques; 2008 [accessed March 2009]. http: //spirit.sourceforge.net/.

[21] Siemens Water and Wastewater Technologies; 2009 [accessed March 2009]. http://www.water. siemens.com.

[22] Chi EH. A Taxonomy of Visualization Techniques Using the Data State Reference Model. In: INFOVIS '00: Proceedings of the IEEE Symposium on Information Vizualization 2000. Washington, DC, USA: IEEE Computer Society; 2000. p. 69-76. citeseer.ist.psu.edu/ chiootaxonomy.html.

[23] Protégé - Free Open Source Ontology Editor; 2009 [accessed March 2009]. http://protege. stanford.edu/.

[24] RSSDB - The RDF Schema Specific DataBase; 2006 [accessed March 2009]. http://139.91.183. $30: 9090 / \mathrm{RDF} / \mathrm{RSSDB} /$.

[25] Einsfeld K, Agne S, Deller M, Ebert A, Klein B, Reuschling C. Dynamic Visualization and Navigation of Semantic Virtual Environments. In: IV '06: Proceedings of the 10th International Conference on Information Visualisation. Washington, DC, USA: IEEE Computer Society; 2006. p. 569-574.

[26] Ware C, Franck G. Evaluating Stereo and Motion Cues for Visualizing Information Nets in Three Dimensions. ACM Transactions on Graphics. 1996;15(2):121-140. citeseer.ist.psu.edu/ ware 96 evaluating.html.

[27] Risch JS. On the role of metaphor in information visualization. The Computing Research Repository (CoRR). 2008;abs/0809.0884.

[28] Gibson JJ. The Ecological Approach to Visual Perception. Lawrence Erlbaum Associates; 1979.

[29] Fraunhofer UMSICHT - Installation of the pilot plant; 2009 [accessed March 2009]. http://www. umsicht. fraunhofer.de/ englisch/presse/bericht.php?titel= 080521_komplett_lieferung $\backslash$.

[30] Jern M, Franzen J. Integrating InfoVis and GeoVis Components. In: IV '07: Proceedings of the 11th International Conference Information Visualization. Washington, DC, USA: IEEE Computer Society; 2007. p. 511-520. 
[31] Roberts JC. Exploratory Visualization with Multiple Linked Views. In: MacEachren A, Kraak MJ, Dykes J, editors. Exploring Geovisualization. Amsterdam: Elseviers; 2004. p. 149-170. http://www.cs. kent.ac.uk/pubs/2004/1822.

[32] Kirner TG, Martins VF. Development of an information visualization tool using virtual reality. In: SAC '00: Proceedings of the 2000 ACM symposium on Applied computing. New York, NY, USA: ACM; 2000. p. 604-606. 